[9] R. Ahlswede and Z. Zhang, "An identity in combinatorial extremal theory," Adr. in Math., vol. 80, no. 2, pp. 137-151. Apr. 1990.

[10] R. Ahlswede and Z. Zhang, "On cloud-antichains and related Configurations," Discrete Math., vol. 85, pp. 225-245, 1990.

[11] A. E. Brouwer, J. B. Shearer, N. J. A. Sloane, and W. D. Smith, "A new table of constant weight codes," IEEE Trans. Inform. Theory. vol. 36 , pp. 1334-1380, Nov. 1990 .

\section{When is the Generalized Likelihood Ratio Test Optimal?}

Ofer Zeitouni, Senior Member, IEEE, Jacob Ziv, Fellow, IEEE, and Neri Merhav, Member, IEEE

\begin{abstract}
The generalized likelihood ratio test (GLRT), which is commonly used in composite hypothesis testing problems, is investigated. Conditions for asymptotic optimality of the GLRT in the NeymanPearson sense are studied and discussed. First, a general necessary and sufficient condition is established, and then based on this, a sufficien condition, which is easier to verify, is derived. A counterexample, where the GLRT is not optimal, is provided as well. A conjecture is stated concerning the optimality of the GLRT for the class of finite-state sources.
\end{abstract}

Index Terms--Hypothesis testing, generalized-likelihood ratio test maximum-likelihood test, error exponent, Neyman-Pearson criterion, large deviations.

\section{INTRODUCTION}

Consider the following prototype classification problem. Let $x^{n}=\left(x_{1}, \cdots, x_{n}\right)$ be a sequence of observations which take values in a finite set $\boldsymbol{X}$ with cardinality $X$. It is assumed that $x^{n}$ has been drawn from a probabilistic source $P$ which is either $P_{0}$ or $P_{1}$, i.e., $P_{0}$ and $P_{1}$ are probability measures on the space $\Omega=X^{2}$ of all infinite sequences and we identify the marginal of $P_{0}$ under the coordinate map $\omega \rightarrow x^{n}$. The classification problem is that of deciding, upon observing $x^{n}$, whether the true underlying source is $P_{0}$ or $P_{1}$. Throughout the sequel, $P_{i}\left(x^{n}\right), i=0,1$, will denote the probability of a string $x^{n}$ under $P_{i}$. Similarly, $P_{i}(F)$ will denote the probability of an event $F$ under either source. A decision rule is a subset $\Lambda_{n}$ of the sample space $\boldsymbol{X}^{n}$ such that if $x^{n} \in \Lambda_{n}$, then $x^{n}$ is classified as being drawn from $P=P_{1}$, otherwise it is classified as $P=P_{0}$. The probability of error of the first kind (false alarm), associated with $\Lambda_{n}$, is defined as $P_{0}\left(\Lambda_{n}\right)$, i,e., the probability of deciding $P=P_{1}$ while $P_{0}$ is the true source. Similarly, the probability of error of the second kind (misdetection) is defined as $P_{1}\left(\bar{\Lambda}_{n}\right)$, where $\bar{\Lambda}_{n}$ is the set complementary to $\Lambda_{n}$.

For known sources $P_{0}$ and $P_{1}$, the classical Nevman-Pearson approach [1] suggests the following optimality criterion: Among all decision rules $\Lambda_{n}$ yiclding

$$
P_{0}\left(\Lambda_{n}\right) \leq 2^{-\lambda n},
$$

for a given $\lambda>0$, find the one which minimizes $P_{1}\left(\bar{\Lambda}_{n}\right)$. It is well known that the solution to this problem is given by the

Manuscript received September 18, 1990. This work was presented at the IEEE International Symposium on Information Theory, Budapest, Hungary, June 24-28, 1991. This work was performed in part while the authors were visiting AT \& T Bell Laboratories, Murray Hill, NJ

The authors are with the Department of Electrical Engineering, Technion-Israel Institute of Technology, Haifa 32000, Israel.

IEEE Log Number 9200885. likelihood ratio test (LRT), i.c.

$$
\Lambda_{n}^{\mathrm{LRT}}=\left\{x^{n}: \frac{1}{n} \log P_{1}\left(x^{n}\right)-\frac{1}{n} \log P_{01}\left(x^{n}\right) \geq T_{n}(\lambda)\right\},
$$

where logarithms throughout the correspondence will be taken to the base 2 and the threshold function $T_{n}(\lambda)$, defined such that $P_{0}\left(\Lambda_{n}^{\mathrm{LRT}}\right)=2^{-\lambda n}$, depends, in general, also on $n, \lambda, P_{0}$, and $P_{1}$.

Suppose next that $P_{0}$ is still known but $P_{1}$ is unknown except that it is a member of some subclass $\boldsymbol{P}$ of the stationary and ergodic sources, namely, a simple hypothesis $P=P_{0}$ is tested against a composite alternative $P \in \boldsymbol{P}$. Clearly, in this case, the LRT (1.2) is no longer applicable. It is common to use, in this situation, the generalized-likelihood ratio test (GLRT) [2, p. 1, pp. 86-96], given by

$$
\Lambda_{n}^{\mathrm{GITT}}=\left\{x^{n}: \frac{1}{n} \log \sup _{P_{1} \in P} P_{1}\left(x^{n}\right)-\frac{1}{n} \log P_{0}\left(x^{n}\right) \geq T_{n}(\lambda)\right\} .
$$

In [3]-[6], the GLRT with $T_{n}(\lambda)=\lambda$ was investigated in several concrete composite hypotheses testing problems, where $\boldsymbol{P}$ was the subclass of all Markov sources up to a given order (including zero order, i.e., memoryless sources). In [7], more generally, a parametric class $\boldsymbol{P}$ from an exponential family was assumed. In fact, all these studies have shown that the GLRT is asymptotically optimal under a modified version of the Neyman-Pearson criterion, defined as follows.

$M$ : Among all sequences of decision rules $\left\{\Lambda_{n}\right\}_{n \geq 1}$ that do not depend on the unknown $P_{1}$ and at the same time satisfy

$$
\limsup _{n \rightarrow \infty} \frac{1}{n} \log P_{0}\left(\Lambda_{n}\right)<-\lambda
$$

select a sequence that maximizes the second kind error exponent, $-\lim \sup _{n \rightarrow \times} n^{-1} \log P_{1}\left(\bar{\Lambda}_{n}\right)$, uniformly for all $P_{1} \in P$.

In other words, the GLRT (1.3), which is independent of $P_{1}$, performs in [3]-[7] asymptotically as well as the optimal LRT (1.2), which in turn depends upon $P_{1}$. However, in [8], where the class $\boldsymbol{P}$ of alternatives was extended to the set of all finite-state (FS) sources with no more than $S$ states, it was not the GLRT that was proved asymptotically optimal, but a generalized version of a test proposed by Hoeffding (sec [9] and Lemma 1 below), where practical implementation turned out to be simplified by the use of the Lempel-Ziv (LZ) algorithm [10]. However, it is not clear from the results of [8] whether the GLRT is still optimal in this case.

In light of these results, the goal of this correspondence is to study relations between the GLRT and the optimal test under the criterion $M$ previously defined. In particular, we are interested in establishing conditions on the class $\boldsymbol{P}$ under which the GLRT is asymptotically optimal in that sense. We first derive a necessary and sufficient condition for asymptotic optimality of the GLRT. Since this condition is, in general, difficult to check, we further derive a sufficient condition, which is easier to verify, and demonstrate that it holds in many interesting special cases, including [3]--[7] as well as other cases. On the other hand, we provide a countercxample to demonstrate that the GLRT is not always asymptotically optimal. The question whether the GLRT is asymptotically optimal in the case that $\boldsymbol{P}$ is the set of all FS 
sources [8], remains open, although we conjecture that the answer is positive, cf., the remark at the end of Section IV.

As a final remark, we point out that although merely finitealphabet processes are considered here, the results are readily extended to more general alphabets if one replaces combinatorial bounds by related large deviations bounds and modifies the optimality criterion appropriately. The interested reader is referred to [11] for a precise description of this extension. Also, by using this approach, one may also use the empirical process (provided that $\boldsymbol{P}$ is a set with sufficiently "nice" ergodic properties) and thus, bypass the Markov approximation approach that is used here (see (2.1) and (2.2)).

\section{Preliminaries}

We shall assume that every source in $\boldsymbol{P}$ can be approximated, to an arbitrary degree of accuracy, by a Markov source of a sufficiently high-order $l$. Specifically, for every source $P \in P$ and an infinite string $x=\left(x_{1}, x_{2}, \cdots\right)$ define

$$
\begin{array}{r}
\rho_{l}(x)=\sup _{n>l}\left|\log P\left(x_{n} \mid x^{n-1}\right)-\log P\left(x_{n} \mid x_{n-l}^{n-1}\right)\right|, \\
\quad l=1,2, \cdots,
\end{array}
$$

where $x_{i}^{j}$ denotes the segment $\left(x_{i}, x_{i+1}, \cdots, x_{j}\right)$ for $j>i$, $P\left(x_{n} \mid x^{n-1}\right)$ is the conditional probability of $x_{n}$ given the entire past, and $P\left(x_{n} \mid x_{n-1}^{n-1}\right)$ is the conditional probability of $x_{n}$ given the $l$ preceding letters. It will be assumed that for every $\varepsilon>0$ there exists a sufficiently large $l$ such that $\rho_{l}(x) \leq \varepsilon$ almost surely. This is equivalent to the condition that for every string $x^{n}$ with nonzero probability,

$$
2^{-\varepsilon n} \prod_{i=1}^{n} P\left(x_{i} \mid x_{i-1}^{i-1}\right) \leq P\left(x^{n}\right) \leq 2^{\varepsilon n} \prod_{i=1}^{n} P\left(x_{i} \mid x_{i-1}^{i-1}\right) .
$$

Since $\varepsilon$ is assumed independent of $x^{n},(2.2)$ tells us we can approximate $P$ by an $l$ th-order Markov process at the expense of degrading the exponential of rate of the second kind error probability, namely $-\lim \sup _{n \rightarrow x} \log P_{1}\left(\bar{\Lambda}_{n}\right)$, by no more than $\varepsilon$. Therefore, we shall throughout consider sources in $\boldsymbol{P}$ as stationary, ergodic, $l$ th-order Markov sources, keeping in mind that the discussion next will focus, in the general case, on $\varepsilon$-optimal rather than optimal error exponents.

Remark: Rather than using $l$ th-order Markov sources for approximating sources in $\boldsymbol{P}$ one may use, more generally, unifilar FS sources having sufficiently many states (see Appendix) with a straightforward extension of (2.1), (2.2), and the forthcoming derivation. This is advantageous if one wishes to approximate general FS sources [8] as demonstrated in the Appendix. Note, however, that although the GLRT is asymptotically optimal, as mentioned earlier, when $\boldsymbol{P}$ is the class of all $l$ th order Markov measures or the class of all unifilar FS sources with $X^{l}$ states, it is not necessarily so when $\boldsymbol{P}$ is the subset of sources, in this class, which approximate (in the sense of (2.2)) all general FS sources with a given number of states.

Let $s_{i}=x_{i-1}^{i-1}$ denote the state at time instant $i$. For simplicity, we shall assume that the initial state $s_{1}=x_{1-1}^{0}$ is fixed. Define the empirical joint probability of a letter $u \in X$ and a state $\nu \in \boldsymbol{X}^{l}$ as

$$
q_{x}^{n}(u, \nu)=\frac{1}{n} \sum_{i=1}^{n} \delta\left(x_{i}, u, s_{i}, \nu\right)
$$

where $\delta\left(x_{i}, u, s_{i}, \nu\right)$ is the indicator function for $x_{i}=u$ jointly with $s_{i}=\nu$. The empirical distribution $Q_{x}^{n}$ is defined as the

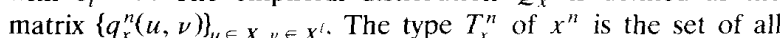
strings $y^{n} \in X^{n}$ with $Q_{v}^{n}=Q_{x}^{n}$. The empirical conditional prob- ability of a letter $u$ given a state $\nu$ is defined as

$$
q_{x}^{n}(u \mid \nu)=\frac{q_{x}^{n}(u, \nu)}{\sum_{u \in X} q_{x}^{n}(u, \nu)},
$$

with the convention that if the denominator (and hence, also the numerator) is zero, then $q_{x}^{n}(u \mid \nu)$ is set to zero as well. Next, define the empirical conditional entropy associated with $x^{n}$ as

$$
H\left(Q_{x}^{n}\right)=-\sum_{u \in X} \sum_{\nu \in X^{\prime}} q_{x}^{n}(u, \nu) \log q_{x}^{n}(u \mid \nu),
$$

and the divergence between $Q_{x}^{\prime \prime}$ and the source $P$ as

$$
D\left(Q_{x}^{n} \| P\right)=\sum_{u \in X} \sum_{\nu \in X^{\prime}} q_{x}^{n}(u, \nu) \log \frac{q_{x}^{n}(u \mid \nu)}{P(u \mid \nu)},
$$

where $P(u \mid \nu)$ is the conditional probability of $x_{i}=u$ given that $s_{i}=\nu$.

The following lemma, which is a straightforward extension of a result due to Hoeffding [9], proposes an asymptotically optimal sequence of decision rules under the criterion $M$ defined in Section I. This lemma will serve as a basic tool for examining the GLRT.

Lemma 1: The sequence of decision rules $\left\{\Lambda_{n}^{*}\right\}_{n \geq 1}$, where

$$
\Lambda_{n}^{*}=\left\{x^{n}: D\left(Q_{x}^{n} \| P_{0}\right) \geq \lambda\right\},
$$

satisfies the constraint

$$
\limsup _{n \rightarrow \infty} \frac{1}{n} \log P_{0}\left(\Lambda_{n}^{*}\right) \leq-\lambda,
$$

and at the same time maximizes, among all sequences of decision rules satisfying (1.4), the second kind error exponent $-\lim$ $\sup _{n \rightarrow \infty} n^{-1} \log P_{1}\left(\bar{\Lambda}_{n}\right)$ for all $P_{1} \in \boldsymbol{P}$.

Proof: To prove (2.8), first observe that the membership of a string $x^{n}$ in $\Lambda_{n}^{*}$ depends only on its empirical distribution (type). Hence,

$$
P_{0}\left(\Lambda_{n}^{*}\right)=\sum_{T_{i}^{n} \subset \Lambda_{n}^{*}} P_{0}\left(T_{x}^{n}\right) .
$$

From $[4$, Lemma 1] we find that

$$
\left|\frac{1}{n} \log P_{0}\left(T_{x}^{n}\right)+D\left(Q_{x}^{n} \| P_{0}\right)\right| \leq \varepsilon_{n},
$$

where $\varepsilon_{n}=O\left(n^{-1} \log n\right)$ independently of $x^{n}$. Furthermore, the total number of distinct types $T_{x}^{n}$ in $X^{n}$ never exceeds $(n+1)^{X^{2 i}}$ [12, p. 434], where $X$ is the cardinality of the source alphabet $X$. Combining this fact with (2.9) and (2.10), we have for every $\varepsilon>0$,

$$
\begin{aligned}
P_{0}\left(\Lambda_{n}^{*}\right) & \leq(n+1)^{X^{2 \prime}} \max _{T_{*}^{n} \in \Lambda_{n}^{*}} P_{0}\left(T_{x}^{n}\right) \\
& \leq(n+1)^{X^{2 l}} \exp _{2}\left\{-n\left[\min _{T_{n}^{n} \in \Lambda_{n}^{*}} D\left(Q_{x}^{n} \| P_{0}\right)-\varepsilon_{n}\right]\right\} \\
& =\exp _{2}\left\{-n\left[\lambda-\varepsilon_{n}-\frac{X^{2 l}}{n} \log (n+1)\right]\right\} \leq 2^{-n(\lambda-\varepsilon)},
\end{aligned}
$$

where the equality follows from the definition of $\Lambda_{n}^{*}$. This completes the proof of (2.8).

As for the second part of the lemma, since $P_{1}$ is assumed an $l$ th order Markov source, the empirical distribution $Q_{r}^{n}$ serves as sufficient statistics for optimal decision under the criterion $M$ (see also [3]-[7]). Let $\left\{\Lambda_{n}\right\}_{n \geq 1}$ be an arbitrary sequence of 
decision rules satisfying (1.4), where membership in $\Lambda_{n}$ depends only on the empirical distribution. Then, from (1.4), for every $\varepsilon>0$, all large $n$ and any $y^{n} \in \Lambda_{n}$,

$$
\begin{aligned}
2^{-\left(\lambda+\varepsilon_{n}\right) n} & \geq P_{0}\left(\Lambda_{n}\right) \geq P_{0}\left(T_{y}^{n}\right) \\
& \geq \exp _{2}\left\{-n\left[D\left(Q_{y}^{n} \| P_{0}\right)+\varepsilon_{n}\right]\right\},
\end{aligned}
$$

where we have used again (2.10). This means that $y^{n}$ is also in $\Lambda_{n}^{*}$, i.e., $\Lambda_{n} \subseteq \Lambda_{n}^{*}$, hence $P_{1}\left(\bar{\Lambda}_{n}^{*}\right) \leq P_{1}\left(\bar{\Lambda}_{n}\right)$ for every $P_{1} \in P$, completing the proof of Lemma 1 .

\section{Remarks:}

1) An important property of the test (2.7) is that it is independent of the class $\boldsymbol{P}$. Moreover, it is easily replaced by a test based on the LZ algorithm (see, e.g., [3]-[6], [8]).

2) Note that $D\left(Q_{x}^{n} \| P_{0}\right)$ in (2.7) can be rewritten as

$$
D\left(Q_{x}^{n} \| P_{0}\right)=-H\left(Q_{x}^{n}\right)-\frac{1}{n} \log P_{01}\left(x^{n}\right),
$$

where $-H\left(Q_{x}^{n}\right)$, in turn, can be also expressed as

$$
-H\left(Q_{x}^{n}\right)=\frac{1}{n} \log P_{1}\left(x^{n}\right)+D\left(Q_{x}^{n} \| P_{1}\right) .
$$

Thus, if $\boldsymbol{P}$ is sufficiently rich so that

$$
\inf _{P_{1} \in P} D\left(Q_{x}^{n} \| P_{1}\right)=0, \quad \forall x^{n} \in X^{n},
$$

then it follows from (2.14) that $-H\left(Q_{r}^{n}\right)=n^{-1}$ $\sup _{P_{1} \in P} \log P_{1}\left(x^{n}\right)$, in which case the GLRT (1.3) with $T_{n}(\lambda)=\lambda$ coincides with Hoeffding's test (2.7) and hence, it is asymptotically optimal. Indeed, in all cases considered in [3]-[6], $\boldsymbol{l}$ was assumed fixed and $\boldsymbol{P}$ was the entire class of $l$ th-order Markov sources, therefore (2.15) holds trivially. It should be noted again that even if a general FS source can be approximated by a high-order Markov source, the latter might have many more states than that of the former, thus (2.15) may not hold.

In the next section, we demonstrate that even in simpler situations the GLRT is not always asymptotically optimal. Before we present such a counterexample, however, we need a preliminary step.

It can be shown, using a technique similar to the proof of Lemma 1, that for a given $P_{1} \in \boldsymbol{P}$, the second kind error exponent, associated with Hoeffding's test (2.7), is given by

$$
-\lim _{n \rightarrow \infty} \frac{1}{n} \log P_{1}\left(\bar{\Lambda}_{n}^{*}\right)=e(\lambda) \triangleq \inf _{Q \in A} D\left(Q \| P_{1}\right),
$$

where $A$ is the set of all $l$ th-order Markov measures $Q$, defined as

$$
A=\left\{Q: D\left(Q \| P_{0}\right) \leq \lambda\right\} .
$$

The function $e(\lambda)$ is called the error-exponent function [13, p. 123, Definition 4.6.1]. Since $B$ is a convex set and $D\left(\cdot \| P_{1}\right)$ is a strictly convex function, the infimum (2.16) is attained uniquely by a measure $\tilde{Q}$ on the boundary of $A$, i.e., $D\left(\tilde{Q} \| P_{0}\right)=\lambda$. Specifically, by standard Lagrange minimization (see also [13, ch. 4]), we find that $\tilde{Q}$ is of the form

$$
\tilde{Q}(u \mid \nu)=K_{\nu} P_{0}^{s}(u \mid \nu) P_{1}^{1-s}(u \mid \nu), \quad \forall u \in X, \quad \nu \in X^{l},
$$

where $s \in[0,1]$ is chosen such that $D\left(\tilde{Q} \| P_{0}\right)=\lambda$ and $K_{v}=$ $\left[\Sigma_{u \in X} P_{0}^{s}(u \mid \nu) P_{1}^{1-s}(u \mid \nu)\right]^{-1}$ is a normalization factor. It is easy to see that the same measure $\tilde{Q}$ minimizes $D\left(Q \| P_{0}\right)$ over the set

$$
B=\left\{Q: D\left(Q \| P_{1}\right) \leq e(\lambda)\right\} .
$$

Consider now a sequence $\left\{T_{x}^{n}\right\}_{n>1}$ of types with $Q_{x}^{n} \rightarrow Q_{0}$ for some $l$ th order Markov measure $Q_{0}$ in the interior of $B$, i.e., $D\left(Q_{0} \| P_{1}\right)<e(\lambda)$, and suppose that for some sequence of decision rules $\left\{\Lambda_{n}\right\}_{n \geq 1}$, satisfying (1.4), $T_{x}^{n}$ is classified into $P=P_{0}$ for infinitely many values of $n$. Then,

$$
\begin{aligned}
-\limsup _{n \rightarrow \infty} \frac{1}{n} \log P_{1}\left(\bar{\Lambda}_{n}\right) & \leq-\limsup _{n \rightarrow \infty} \frac{1}{n} \log P_{1}\left(T_{x}^{n}\right) \\
& =D\left(Q_{0} \| P_{1}\right)<e(\lambda),
\end{aligned}
$$

which means, in view of (2.16), that this sequence of decision rules is not optimal. We have just proved the following lemma, which provides a necessary condition for optimality of any given sequence of decision rules.

Lemma 2 : Let $\left\{\Lambda_{n}\right\}_{n \geq 1}$ be an asymptotically optimal sequence of decision rules under the criterion $M$, where $Q_{x}^{n}$ is sufficient statistics for $\Lambda_{n}$, and let $\left\{T_{x}^{n}\right\}_{n \geq 1}$ be a sequence of types such that the corresponding sequence of empirical distributions $\left\{Q_{x}^{\prime \prime}\right\}_{n \geq 1}$ has an accumulation point $Q_{0}$ in the interior of $B$ for some $P_{1} \in P$. Then, for all large $n, T_{x}^{n} \subseteq \Lambda_{n}$.

In other words, the lemma states that every string $x^{n}$ whose empirical distribution falls in a "sphere" $B$ with radius $e(\lambda)$, centered at $P_{1}$, must be classified into $P=P_{1}$ for sufficiently large $n$. As an example of an asymptotically optimal sequence of decision rules, other than Hoeffding's test, suppose that $P_{1}$ is known (i.e., $\boldsymbol{P}=\left\{P_{1}\right\}$ and consider the LRT (1.2) with the optimal threshold function. Since both Hoeffding's test and the LRT are asymptotically optimal, in this case, then by comparing the performance of the two tests, it is easy to show that the threshold function $T_{n}(\lambda)$ of the optimal LRT tends to a limit $T(\lambda)$ given by

$$
T(\lambda)=\lambda-e(\lambda) .
$$

This relation can be also obtained from a straightforward extension of [13, p. 120, Theorem 4.5.2].

\section{A COUNTEREXAMPLE}

Let $P_{0}$ be a binary memoryless (Bernoulli) source with letter probabilities $P_{0}(" 0 ")=P_{0}(" 1 ")=0.5$. Let $\boldsymbol{P}$ be the class of all Bernoulli sources $P_{1}$ where the parameter $\theta \triangleq P_{1}($ " 0 ") is either larger than 0.7 or smaller than 0.2 . The idea behind this choice of an asymmetric $\boldsymbol{P}$, is that the "distances" of its two parts, $\{\theta$ : $\theta \leq 0.2\}$ and $\{\theta: \theta \geq 0.7\}$, from $\theta=0.5$, which corresponds to $P_{1)}$, are different and hence a fixed threshold function $T_{n}(\lambda)$ cannot fit both parts of $\boldsymbol{P}$. Throughout this section, we shall denote a Bernoulli source with parameter $\theta, 0 \leq \theta \leq 1$, by $P^{\theta}$. Since all sources involved are memoryless, the relative frequencies of letters $q_{x}^{\prime \prime}(u) \triangleq n^{-1} \sum_{l=1}^{n} \delta\left(x_{i}, u\right), u=$ "0", "1", are sufficient statistics in this case.

We now show that for $\lambda=D\left(P^{0.4} \| P^{0.5}\right)=D\left(P^{0.6} \| P^{0.5}\right)$ there is no threshold value $T_{n}(\lambda)$ for which the GLRT is asymptotically optimal. Define

$$
\begin{aligned}
& \lambda_{1}=D\left(P^{0.6} \| P^{0.7}\right), \\
& \lambda_{2}=D\left(P^{0.4} \| P^{0.2}\right) .
\end{aligned}
$$

Assume first, that $T_{n}(\lambda) \leq \lambda-\lambda_{1}$ and consider the type of strings $x^{n}$ for which $q_{x}^{n}($ " 0 ") $=0.6$. For the GLRT to satisfy the constraint (1.4), this type must be classified into $P_{0}$. Since 
$\sup _{P_{1} \in P} P_{1}\left(x^{n}\right)=P^{0.7}\left(x^{n}\right)$, as can be easily shown, we have that

$$
\begin{aligned}
& \frac{1}{n} \log \sup _{P_{1}} \in P \\
& \quad P_{1}\left(x^{n}\right)-\frac{1}{n} \log P_{0}\left(x^{n}\right) \\
& \quad=\frac{1}{n} \log P^{0.7}\left(x^{n}\right)-\frac{1}{n} \log P_{0}\left(x^{n}\right) \\
& \quad=D\left(P^{0.6} \| P^{0.5}\right)-D\left(P^{0.6} \| P^{0.7}\right)=\lambda-\lambda_{1} \geq T_{n}(\lambda),
\end{aligned}
$$

which means that the GLRT classifies $x^{n}$ into $P=P_{1}$ and hence contradicts the previous requirement that $x^{n}$ should be classified to $P_{0}$.

Assume next, that $T_{n}(\lambda)>\lambda-\lambda_{1}$, and consider a string $x^{n}$ for which $q_{x}^{n(" 0 ")}=0.35$. Since $D\left(P^{0.35} \| P_{01}\right)>\lambda$ and $P^{0.35}$ lies "between" $P_{1}=P^{0.2} \in P_{1}$ and $P_{0}=P^{0.5}$, then $D\left(P^{0.35} \|\right.$ $\left.P^{0.2}\right)<e(\lambda)$, and hence by Lemma $2, x^{n}$ must be classified into $P=P_{1}$. Since $D\left(P^{0.35} \| P^{0.2}\right)<D\left(P^{0.35} \| P^{0.7}\right)$, this implies that $\sup _{P_{1} \in P} P_{1}\left(x^{n}\right)=P^{0.2}\left(x^{n}\right)$. Finally, since

$$
D\left(P^{0.35} \| P^{0.5}\right)-D\left(P^{0.35} \| P^{0.2}\right)<\lambda-\lambda_{1} .
$$

as can one easily verify, we find that

$$
\begin{aligned}
& \frac{1}{n} \log \sup _{P_{1} \in P} P_{1}\left(x^{n}\right)-\frac{1}{n} \log P_{0}\left(x^{n}\right) \\
& \quad=D\left(P^{0.35} \| P^{0.5}\right)-D\left(P^{0.35} \| P^{0.2}\right)<\lambda-\lambda_{1}<T_{n}(\lambda),
\end{aligned}
$$

which implies that the GLRT classifies $x^{n}$ into $P_{0}$ in contrast to the previous requirement that $x^{n}$ should be classified into $P_{1}$.

Since we have contradicted both possibilities $T_{n}(\lambda) \leq \lambda-\lambda_{1}$ and $T_{n}(\lambda)>\lambda-\lambda_{1}$, we have proved the nonexistence of a threshold value for which the GLRT is asymptotically optimal under the criterion $M$.

\section{CONDITIONS FOR ASYMPTOTIC OPTIMALITy OF THE GLRT}

We begin by deriving a necessary and sufficient condition for the asymptotic optimality of the GLRT in the situation described in Section II. For the sake of simplicity and in view of the Remark 2 in Section II, we confine attention to the case $T_{n}(\lambda)=\lambda$.

Theorem 1: Let $\boldsymbol{P} \cup\left\{P_{0}\right\}$ be a subset of the class of stationary, ergodic $l$ th-order Markov sources. Then, the GLRT with a threshold function $T_{n}(\lambda)=\lambda$ is asymptotically optimal under criterion $M$, if and only if for all $P_{1} \in P$,

$$
\inf _{Q \in C} D\left(Q \| P_{1}\right) \geq e(\lambda)
$$

where

$$
C=\left\{Q: D\left(Q \| P_{0}\right)-\inf _{P_{1} \in P} D\left(Q \| P_{1}\right)<\lambda \leq D\left(Q \| P_{0}\right)\right\} .
$$

The interpretation of the theorem is as follows. The right-hand side of (4.1) is the second kind error exponent associated with Hoeffding's test (2.7), which is always optimal by Lemma 1, and we want the second kind error exponent of the GLRT to be as large. The right-hand side of (4.1) corresponds to the exponential rate of the probability of the set $\Lambda_{n}^{*}-\Lambda_{n}^{\mathrm{GLRT}}$, namely, the set of all sequences which are classified to $P_{0}$ by the GLRT but not by Hoeffding's test. If this exponential rate is larger than Hoeffding's exponent, then the contribution of this difference set is exponentially negligible and the two tests provide the same performance. Note that if the simple sufficient condition (2.15) is satisfied, as in the case where $\boldsymbol{P}$ contains all Markov sources of order $l$ or less, then (4.1) holds trivially because $C$ becomes an empty set.

Proof of Theorem 1: We first show that the GLRT with $T_{n}(\lambda)=\lambda$ satisfies (1.4). To this end, it is sufficient to show that $\Lambda_{n}^{\text {GI.RT }} \subseteq \Lambda_{n}^{*}$, as we already know from Lemma 1 that $\Lambda_{n}^{*}$ satisfies (1.4). This, in turn, follows from the following consideration:

$$
\begin{aligned}
& \frac{1}{n} \underset{P_{1} \in P}{\log \sup _{1}} P_{1}\left(x^{n}\right)-\frac{1}{n} \log P_{0}\left(x^{n}\right) \\
& \quad=-H\left(Q_{x}^{n}\right)-\inf _{P_{1} \in \boldsymbol{P}} D\left(Q_{x}^{n} \| P_{1}\right)-\frac{1}{n} \log P_{0}\left(x^{n}\right) \\
& \quad \leq-H\left(Q_{x}^{n}\right)-\frac{1}{n} \log P_{0}\left(x^{n}\right) \\
& \quad=D\left(Q_{x}^{n} \| P_{0}\right),
\end{aligned}
$$

where the inequality follows from the fact that a divergence is nonnegative. Thus, (4.3) tells us that if $x^{n}$ is such that the left-most side exceeds $\lambda$, namely, $x^{n} \in \Lambda_{n}^{\mathrm{GLRT}}$, then the rightmost side cxceeds $\lambda$ as well, i.e., $x^{n} \in \Lambda_{n}^{*}$. This completes the proof that $\Lambda_{n}^{\mathrm{GLRT}} \subseteq \Lambda_{n}^{*}$ or, equivalently, $\bar{\Lambda}_{n}^{*} \subseteq \bar{\Lambda}_{n}^{\mathrm{GLRT}}$.

In view of this fact, in order to examine $P_{1}\left(\bar{\Lambda}_{n}^{G L R T}\right)$, we can decompose this probability as

$$
P_{\mathrm{l}}\left(\bar{\Lambda}_{n}^{\mathrm{GI} \cdot \mathrm{RT}}\right)=P_{\mathrm{l}}\left(\bar{\Lambda}_{n}^{*}\right)+P_{1}\left(\bar{\Lambda}_{n}^{\mathrm{GLRT}} \cap \Lambda_{n}^{*}\right)
$$

The first term on the right-hand side is the second kind error probability associated with Hoeffding's test, which decays exponentially with rate $e(\lambda)$, as mentioned earlier. Similarly, the second term on the right-hand side of (4.4) decays with an exponential rate given by the left-hand side of (4.1), as all sequences in $\bar{\Lambda}_{n}^{\mathrm{Gl} . \mathrm{RT}} \cap \Lambda_{n}^{*}$ have their empirical distributions in $C$. Therefore, we observe that the GLRT is asymptotically optimal, if and only if the exponential rate of the second term of (4.4) is at least as large as that of the first term, which is exactly condition (4.1). This completes the proof of Theorem 1.

The necessary and sufficient condition of Theorem 1 is difficult to check in general. We, therefore, derive a sufficient condition (Theorem 2) for the asymptotic optimality of the GLRT, which is easier to verify.

Theorem 2: Let $\boldsymbol{P} \cup\left\{P_{0}\right\}$ be a subset of the class of stationary, ergodic $l$ th-order Markov sources, and let $\tilde{Q}=$ arg $\min _{Q \in B} D\left(Q \| P_{0}\right)$, where $B$ is defined as in (2.19). If $\tilde{Q} \in \boldsymbol{P}$ for all $P_{1} \in P$, then the GLRT with the threshold function $T_{n}(\lambda)=\lambda$ is asymptotically optimal under criterion $M$.

Since $\tilde{Q}$ is given by an "exponential combination" of $P_{0}$ and $P_{1}$ (see (2.18)), the significance of Theorem 2 is that if $\boldsymbol{P} \cup\left\{P_{0}\right\}$ is closed with respect to such exponential combinations, then the GLRT is asymptotically optimal. It is easy to see that this closure property holds if $\boldsymbol{P}$ is an exponential family of measures as is the case in each of the earlier studies [3]-[7]. This holds true even if one uses statistics of order higher than that of the sufficient statistics (e.g., when $\boldsymbol{P}$ is a subclass of the first-order 
Markov processes but $Q_{x}^{n}$ corresponds to $l>1$ ). In this case, (2.15) is not satisfied, yet $\tilde{Q} \in \boldsymbol{P}$ and hence, the GLRT is asymptotically optimal. This condition holds also for many other subclasses $\boldsymbol{P}$ of the $l$ th-order Markov sources, e.g., the subclass defined by dependence only upon even lags of the process, i.c.,

$$
P\left(x_{i} \mid x^{i-1}\right)=P\left(x_{i} \mid x_{i-2}, x_{i-4}, \cdots, x_{i-1}\right),
$$

where $l$ is assumed even. Again, this class does not satisfy the simple sufficient condition (2.15) because there exist empirical lth-order Markov measures $Q_{x}^{n}$ that are far apart from any Markov measure in the subclass defined by (4.5), e.g., measures $Q_{x}^{n}$ for which $x_{i}$ depends strongly on odd lags $x_{i-1}, x_{i-3}$, etc. For binary memoryless sources, Theorem 2 means convexity of $\boldsymbol{P} \cup\left\{P_{0}\right\}$, which is clearly not the case in the counterexample of Section III. Finally, we point out that since Theorem 2 provides merely a sufficient condition, it is easy to find an example where this condition does not hold and yet the GLRT is asymptotically optimal. A simple example is when $\boldsymbol{P}$ includes only one measure $P_{1}$, in which case the GLRT coincides with the LRT and hence optimal, however, the condition is clearly not satisfied.

Proof of Theorem 2: Since by assumption $\bar{Q} \in \boldsymbol{P}$, then,

$$
D(Q \| \tilde{Q}) \geq \inf _{P_{1} \in \boldsymbol{P}} D\left(Q \| P_{1}\right),
$$

and hence,

$$
\inf _{Q \in C} D\left(Q \| P_{1}\right) \geq \inf _{Q \in D} D\left(Q \| P_{1}\right)
$$

where

$$
D=\left\{Q: D\left(Q \| P_{0}\right)-D(Q \| \tilde{Q})<\lambda \leq D\left(Q \| P_{0}\right)\right\} .
$$

Thus, to prove that (4.1) holds, and hence the GLRT is asymptotically optimal, it is sufficient to show that

$$
\inf _{Q \in D} D\left(Q \| P_{1}\right) \geq \inf _{Q \in A} D\left(Q \| P_{1}\right)=e(\lambda) .
$$

Conversely, assume that (4.9) is false, namely, the measure $\hat{Q} \in D$ that minimizes $D\left(Q \| P_{1}\right)$ on the left-hand side of $(4.10)$ is an interior point of $B$. Then, it follows from [14, Theorem 2.2] that

$$
D\left(\hat{Q} \| P_{0}\right)-D(\hat{Q} \| \tilde{Q}) \geq D\left(\tilde{Q} \| P_{0}\right)=\lambda,
$$

which contradicts the assumption that $\hat{Q} \in D$ and hence, completes the proof of Theorem 2 .

\section{Remarks:}

1) M. Gutman has shown us that a condition somewhat different than that of Theorem 2 is actually both necessary and sufficient. Specifically, referring to the notations of Theorem 2, the GLRT is asymptotically optimal, if and only if

$$
\forall P_{1} \in \boldsymbol{P}, \quad \inf _{P^{\prime} \in \boldsymbol{P}} D\left(\tilde{Q} \| P^{\prime}\right)=0 .
$$

2) Although it has been assumed in this correspondence that $P_{0}$ is completely known, the results can be easily generalized to the case where $P_{0}$ is unknown except that it is in a subset $Q$ of the $l$ th-order Markov sources. In this case, (1.4) is required to hold for all $P_{0} \in Q$, or, equivalently,

$$
\limsup _{n \rightarrow \infty} \frac{1}{n} \log \sup _{P_{n} \in Q} P_{0}\left(\Lambda_{n}\right)<-\lambda .
$$

Similarly, $P_{0}\left(x^{n}\right)$ in the GLRT (1.3) will be replaced by $\sup _{P_{10} \in Q} P_{0}\left(x^{n}\right)$. In all cases studied in [3]-[8], $Q$ was a subset of $\boldsymbol{P}$.

3) We conjecture that for the case where $\boldsymbol{P}$ is the class of all finite-state sources with a given number of states (see Appendix and [8]), the condition of Theorem 2, and hence also the asymptotic optimality of the GLRT, holds true. Although we have not been able to prove it analytically, a computer search over the space $\boldsymbol{P}$ of two-state binary sources, with $l=7$ and $P_{0}$ being a binary memoryless symmetric source (similar to that of Section III), failed to provide a counterexample for which $\tilde{Q}$, as defined in (2.18), falls outside $\boldsymbol{P}$.

\section{APPENDIX}

APProximation of FS SOURCES by UNIFILAR FS SOURCES

An FS source $P$, (also called a hidden Markov source [15],) is characterized by an observed output process $\left(x_{1}, x_{2}, \cdots\right), x_{i} \in X$, and a corresponding unobserved (hidden) finite-alphabet state process $\left(s_{1}, s_{2}, \cdots\right), s_{i} \in S$, which are jointly Markov, i.e.,

$$
P\left(x^{n}, s^{n+1}\right)=\prod_{i=1}^{n} p\left(x_{i}, s_{i+1} \mid s_{i}\right),
$$

where we shall assume that the initial state $s_{1}=\sigma$ is fixed. If, in addition,

$$
s_{i+1}=g\left(x_{i}, s_{i}\right)
$$

with probability 1 , for some mapping $g: X \times S \rightarrow S$, then (A.1) is referred to as an unifilar FS source. In this case, the state process can be reconstructed recursively from the observation process using (A.2). An important special case of a unifilar FS source is an $l$ th-order Markov Source where $s_{i}=x_{i-l}^{i-1}$.

We show that a general FS source (A.1) with strictly positive transition probabilities $\{p(u, \nu \mid w\}\}_{u \in X, v, w \in S}$ can be approximated by a unifilar FS source with a particular choice of $g$ and sufficiently many states. Suppose that $p(u, v \mid w) \geq \delta>0$ for all $u \in X, \nu, w \in S$, and hence,

$$
\delta \cdot p(u, \nu \mid \sigma) \leq p(u, \nu \mid w) \leq \delta^{-1} p(u, \nu \mid \sigma) .
$$

Assume further that $l$ divides $n$ and parse $x^{n}$ into $n / l$ nonoverlapping blocks of length $l$. Then,

$$
\begin{aligned}
P\left(x^{n}\right)= & \sum_{s_{2}^{n+1}} \prod_{i=1}^{n} p\left(x_{i}, s_{i+1} \mid s_{i}\right) \\
= & \sum_{s_{2}^{n}+1} \prod_{j=0}^{n / l-1} \prod_{i=1}^{l} p\left(x_{j l+i}, s_{j l+i+1} \mid s_{j l+i}\right) \\
\geq & \sum_{s_{2}^{n+1}} \prod_{j=0}^{n / l-1} \delta \cdot p\left(x_{j l+1}, s_{j l+2} \mid \sigma\right) \\
& \cdot \prod_{i=2}^{l} p\left(x_{j l+i}, s_{j l+i+1} \mid s_{j l+i}\right) \\
= & \delta^{n / l} \prod_{j=0}^{n / l-1} \sum_{s_{j l+1+1}} p\left(x_{j l+1}, s_{j l+2} \mid \sigma\right) \\
& \cdot \prod_{i=2}^{l} p\left(x_{j l+i}, s_{j l+i+1} \mid s_{j l+i}\right)
\end{aligned}
$$




$$
=2^{-n l^{-1} \log (1 / \delta)} \prod_{j=0}^{n / l-1} P\left(x_{j l+1}^{j l+l}\right) .
$$

In a similar manner we obtain

$$
P\left(x^{n}\right) \leq 2^{n l^{-1}} \log (1 / \delta) \prod_{j=0}^{n / l-1} P\left(x_{j l+1}^{j l+l}\right),
$$

which together with (A.3) means that (A.1) can be approximated by a block memoryless source of $l$-tuples (see also [16, Appendix]) defined by

$$
\tilde{P}\left(x^{n}\right)=\prod_{j=0}^{n / l-1} P\left(x_{j l+1}^{j l+l}\right)=\prod_{j=0}^{n / l-1} \prod_{i=1}^{l} P\left(x_{j l+i} \mid x_{j l+1}^{j l+i-1}\right) .
$$

Under the measure $\tilde{P}, x_{i}$ depends at most on the $l$ preceding letters $x_{i-1}^{i-1}$ and the induced conditional probability $P\left(x_{i} \mid x_{i-l}^{i-1}\right)$, in turn, depends on the position of $i$ with respect to the block endpoints, i.e., on $i \bmod l$. Hence, (A.5) can be described as a unifilar FS source with no more than $l \cdot X^{l}$ states ( $X$ is the alphabet size), where the state variable $\tilde{s}_{i}$ consists of the $l$ preceding letters and a modulo-l time counter $c_{i}$, i.e., $\tilde{s}_{i}=$ $\left(x_{i-l}^{i-1}, c_{i}\right)$. Thus, from (A.3) and (A.4) we find that if $l>$ $\varepsilon^{-1} \log (1 / \delta)$, then with probability 1 ,

$$
2^{-n \varepsilon} \prod_{i=1}^{n} P\left(x_{i} \mid \tilde{s}_{i}\right) \leq P\left(x^{n}\right) \leq 2^{n \varepsilon} \prod_{i=1}^{n} P\left(x_{i} \mid \tilde{s}_{i}\right),
$$

which is similar to (2.2) with the generalization that $x_{i-l}^{i-1}$ is replaced by $\tilde{s}_{i}$.

\section{REFERENCES}

[1] J. Neyman and E. S. Pearson, "On the use and interpretation of certain test criteria for purposes of statistical inference," Biometrika, vol. 20-A, pp. 175-240, 264-299, 1928.

[2] H. Van Trees, Detection, Estimation, and Modulation Theory. New York: Wiley, 1968.

[3] J. Ziv, "On classification with empirically observed statistics and universal data compression," IEEE Trans. Inform. Theory, vol. 34, pp. 278-286, Mar. 1988.

[4] M. Gutman, "Asymptotically optimal classification for multiple tests with empirically observed statistics," IEEE Trans. Inform. Theory, vol. 35, pp. 401-408, Mar. 1989.

[5] - "On tests for independence, tests for randomness and universal data compression," submitted for publication.

[6] N. Merhav, M. Gutman, and J. Ziv, "On the estimation of the order of a Markov chain and universal data compression," IEEE Trans. Inform. Theory, vol. 35, pp. 1014-1019, Sept. 1989.

[7] N. Merhav, "The estimation of the model order in exponential families," IEEE Trans. Inform. Theory, vol. 35, pp. 1109-1114, Sept. 1989.

[8] J. Ziv and N. Merhav, "Estimating the number of states of a finite-state source," IEEE Trans. Inform. Theory, vol. 38, pp. 61-65, Jan. 1992.

[9] W. Hoeffding, "Asymptotically optimal tests for multinomial distributions," Ann. Math. Statist., vol. 36, pp. 369-401, 1965.

[10] J. Ziv and A. Lempel, "Compression of individual sequences via variable-rate coding," IEEE Trans. Inform. Theory, vol. IT-24, pp. 530-536, Sept. 1978.

[11] O. Zeitouni and M. Gutman, "On universal hypotheses testing via large deviations," IEEE Trans. Inform. Theory, vol. 37, pp. 285-290, Mar. 1991; correction, vol. 37, p. 698, May 1991.

[12] L. D. Davisson, G. Longo, and A. Sgarro, "The error exponent for the noiseless encoding of finite ergodic Markov sources," IEEE Trans. Inform. Theory, vol. IT-27, pp. 431-438, July 1981.

[13] R. E. Blahut, Principles and Practice of Information Theory. Reading, MA: Addison-Wesley, 1987

[14] I. Csiszár, "I-divergence geometry of probability distributions and minimization problems," Ann. Probab., vol. 3, no. 1, pp. 146-158, 1975.

[15] L. R. Rabiner, "A tutorial on hidden Markov models and selected applications in speech recognition," Proc. IEEE, vol. 77 , pp. 257-286, Feb. 1989.
[16] N. Merhav and Y. Ephraim, "A Bayesian classification approach with application to speech recognition," IEEE Trans. Signal Processing, vol. 39, pp. 2157-2166, Oct. 1991.

\section{Recursively Indexed Quantization of Memoryless Sources}

Khalid Sayood, Member, IEEE, and Sangsin Na, Member, IEEE

Abstract-A recursively indexed scalar quantizer that performs as well as high-dimensional vector quantizers for several important sources, without the attendant complexity is presented.

Index Terms-Quantization, source coding.

\section{INTRODUCTION}

Consider the source coding scenario of Fig. 1. A $k$-dimensional $N$-point (or output vector) quantizer $Q$ is a mapping of the $k$-dimensional Euclidean space $\boldsymbol{R}^{k}$ to a set of $k$-dimensional quantization vectors $\left\{y_{0}, y_{1}, \cdots, y_{N-1}\right\}$. The quantization rule, denoted by $Q$, is

$$
Q(x)=y_{j}, \quad \text { if } x \in S_{j},
$$

where $\left\{S_{j}: j=0,1, \cdots, N-1\right\}$ is a partition of $\boldsymbol{R}^{k}$, and $\left\{S_{j}\right\}$ are called the quantization regions. A fixed-to-fixed length binary encoder is a mapping from the set $\{0,1, \cdots, N-1\}$ of the indices of the quantization vectors to a set of binary sequences of length $\left\lceil\log _{2} N\right\rceil$, where $[x]$ is the smallest integer not exceeded by $x$. A fixed-to-variable length binary encoder is a mapping from the set $\{0,1, \cdots, N-1\}$ of the indices of the quantization vectors to a set of binary sequences of varying lengths. The quantizer operates in the following fashion: in the first operation cycle the quantizer $Q$ takes the first $k$ source symbols $X_{1}, X_{2}, \cdots, X_{k}$ and produces the corresponding reproduction symbols $Y_{1}, Y_{2}, \cdots, Y_{k}$. It then enters into the next operation cycle, where it takes the next $k$ source symbols $X_{k+1}, X_{k+2}, \cdots, X_{2 k}$ to produce $Y_{k+1}$, $Y_{k+2}, \cdots, Y_{2 k}$. The binary encoder takes a block of indices of reproduction vectors and encodes it into a binary sequence for transmission over a noiseless channel or for storage. At the receiver, the received binary sequence is decoded into a sequence of reproduction indices, which are mapped back to the reproduction vectors via stored quantization vectors.

The performance of a source coding scheme is often measured by its rate and distortion. The rate is the average number of binary digits used for representing a source symbol. When a fixed-to-fixed length binary encoder is used, the rate is $(1 / k)\left[\log _{2} N\right\rceil$ where $N$ is the size of the reproduction alphabet and $k$ is the dimension of the input vectors. The distortion is in some sense a measure of the average closeness of the reproduction sequence to the source sequence. In this correspondence, we will measure the distortion by $(1 / k) E\left\{\|\boldsymbol{X}-Q(X)\|^{2}\right\}$, where the expectation $E$ is taken with respect to the $k$-dimensional source distribution $p(x)$ and where $\|\cdot\|$ denotes the $L^{2}$ norm.

Manuscript received January 31, 1991; revised January 17, 1992. This work was supported by the NASA Lewis Research Center under Grant NAG3-806. This work was presented in part at the Asilomar Conference on Signals, Systems, and Computers, Pacific Grove, CA, November 1990.

K. Sayood is with the Department of Electrical Engineering and the Center for Communication and Information Science, University of $\mathrm{Ne}-$ braska, 209 N. WSEC, Lincoln, NE 68588-0511.

$\mathrm{S}$. Na is with the Department of Electronic Engineering, Ajou University, 5 Wonchun-Dong, Suwon, Korea.

IEEE Log Number 9200887

$0018-9448 / 92 \$ 03.00$ (C) 1992 IEEE 